\title{
Electron and Ion Stagnation at the Collision Front Between Two Laser Produced Plasmas
}

\author{
P Hough ${ }^{1}$, C McLoughin ${ }^{1}$, T J Kelly ${ }^{1}$, P Hayden ${ }^{1}$, S S Harilal ${ }^{2}$, \\ J P Mosnier ${ }^{1}$, J T Costello ${ }^{1}$ \\ ${ }^{1}$ National Centre for Plasma Science and Technology and School of Physical Sciences, \\ Dublin City University, Glasnevin, Dublin 9. \\ ${ }^{2}$ School of Nuclear Engineering, Purdue University, 400 Central Drive, West \\ Lafayette, IN 40707, USA. \\ E-mail: padraighough@gmail.com
}

\begin{abstract}
We report results from a combined optical interferometric and spectrally resolved imaging study on colliding laser produced aluminium plasmas. A Nomarski interferometer was used to probe the spatio-temporal distribution of electron densities at the collision front. Analysis of the resulting interferograms reveals the formation and evolution of a localised electron density feature with a well defined profile reminiscent of a stagnation layer. Electron stagnation begins at a time delay of $10 \mathrm{~ns}$ after the peak of the plasma generating laser pulse. The peak electron density was found to exceed $10^{19} \mathrm{~cm}^{-3}$ and the layer remained well defined up to a time delay of $c a .100 \mathrm{~ns}$. Temporally and spectrally resolved optical imaging was also undertaken, to compare the $\mathrm{Al}^{+}$ion distribution with that of the 2D electron density profile. This revealed nascent stagnation of singly charged ions at a delay time of $20 \mathrm{~ns}$. We attribute these results to the effects of space charge separation in the seed plasma plumes.
\end{abstract}

PACS numbers: $52.30 \mathrm{Ex}, 52.38 \mathrm{Mf}$

Submitted to: Journal of Physics D: Applied Physics 


\section{Introduction}

Laser produced plasmas, formed when a high power pulsed laser is focussed onto a solid density target, have been the subject of considerable attention since their discovery in 1960's [1]. Applications include Pulsed Laser Deposition (PLD) [2], extreme ultraviolet (EUV) light sources [3] and ion accelerators [4]. Colliding laser produced plasmas have been a largely unexplored and an especially unexploited research domain until quite recent times despite the fact that first experiments can be traced back to the early 1970s [5]. Furthermore, most experiments to date have focussed on very high energy density plasmas in large scale experiments [6, 7 and 8] rather than on table-top scale experiments which are more likely to impact routine and wide scale applications in materials science, analytical science, EUV and X-ray light sources. For example, it is already known from experiments on double pulse plasma generation that preheated plasmas, formed by one laser pulse, can be engineered to optimize absorption of a second delayed pulse and yield radiation gain [9, 10 and 11]. As the stagnation layer is itself a low temperature plasma, it could act as an optimal target for these and other applications. Much of the motivation for the study of energetic colliding plasmas comes from experiments on indirect drive fusion where multiple plasma x'ray sources are used to drive fuel cell implosion with much reduced losses due to instabilities. Clearly the hohlraum target cavity hosts multiple colliding plasmas [12] and so the elucidation of the fundamental physics underlying their interactions (using e.g. time resolved optical and x-ray diagnostics[13]) is critically important. Another domain where colliding plasmas are having impact is that of shock wave physics, especially in laboratory simulation of astrophysical plasmas [14].

When two plasmas collide, the outcome usually lies somewhere between two extreme scenarios where the plasmas can either completely interpenetrate or decelerate rapidly at the collision front, i.e., they can stagnate. In the interpenetration phase the plasmas stream through each other, the main interaction amounting to binary collisions. In the case of hard stagnation, rapid accumulation of plasma material at the collision front leads to the formation of a dense layer of material between the two plasmas, the so called stagnation layer [15]. However, even in experiments where well defined stagnation layers are formed, Bosch et al. [16] have shown that the stagnation phase can be preceded by interpenetration as the prompt dilute plasma fronts have not reached the critical density needed to initiate stagnation. For this reason, although some work has already been conducted on colliding plasmas [17], there is still much to be learned regarding the stagnation process itself. Therefore, the study of plasma plume constituents and their interactions before, as well as during the early stages of stagnation layer formation is critical to obtaining a more complete picture of the myriad of physical processes underpinning the plasma-plasma collisions [18]. This is the primary motivation for our experimental investigation.

In this paper we report a study of the interaction region between two colliding laser produced plasma plumes employing both spectrally resolved imaging and spatially 
resolved interferometry to help us to track both singly charged ions and electrons respectively. We have also obtained broadband images, covering the $350-900 \mathrm{~nm}$ range, representative of whole plasma species emission. By these combined means we can map the different constituents (electrons and ions and plasma) in space and time. Our motivation here is to elucidate the plasma conditions which pertain just before and during stagnation layer formation. Our focus is on small scale nanosecond laser systems with energies $\leq 1 \mathrm{~J}$, which are most likely to have greatest impact in laser ablation and short wavelength light sources. We are especially interested in the electron distribution at the collision front since electrons from the rapidly expanding seed plasma plumes are known to lead the ions very slightly [19], at least within the limits set by ambipolar diffusion, and so will be the first to interact at the collision front and potentially impact subsequent plasma-plasma interactions in that vicinity. We note here that EUV and optical interferometry have been used in the past in the study of colliding plasmas [20] and blast waves in low density cluster sources [21]. In these cases the seeds were formed at very high energy density in contrast to the relatively low temperature seed plasmas that are the subject of this study.

\section{Experiment}

The optical system used to split and focus the single laser beam is similar to that used by Harilal et al. [22]. This is shown schematically in Figure 1. The seed plasma plumes were created by dividing a $1064 \mathrm{~nm}$ wavelength, $600 \mathrm{~mJ}, 6 \mathrm{~ns}$ full width at half max (FWHM) laser pulse into two parts with the aid of a $0.5^{\circ}$ wedge prism and focusing it onto two spots, each of $100 \mu \mathrm{m}$ diameter, by means of a $f / 6$ plano-convex lens located at $45^{\circ}$ to the target normal. The peak irradiance at each spot was $6 \times 10^{11} \mathrm{Wcm}^{-2}$. The seed plasmas had a separation of $1.3 \mathrm{~mm}$ and the target was a flat slab of $99.9 \%$ pure aluminium which was mounted on a high precision in-vacuum motorised X-Z stage so that the target could be moved to reveal a new surface after each shot. The pressure in the interaction chamber was maintained at better than $1 \times 10^{-5}$ mbar during all experiments reported here.

Under appropriate conditions, a stagnation layer forms at the interface between the two laterally colliding plasmas. Spectrally resolved optical imaging of the colliding plasmas reveals the 2-D spatial and temporal evolution of singly charged ions in the seed plasmas and stagnation layer. We imaged the $\mathrm{Al}^{+}$distribution with the aid of a framing camera with a shutter time of 3 ns and the electron distribution using time resolved interferometry. The framing camera setup used was the same as that described by Doria et al. [23] and so we give only salient details here. The camera, an intensified charged coupled device (or ICCD), comprised a gated intensifier coupled to front illuminated (CCD) camera via a high quality relay lens system. It was supplied by Andor Technology Ltd (Model No. DH5H7) and had $512 \times 512$ pixels with a pixel area of $24 \mu \mathrm{m} \times 24 \mu \mathrm{m}$ yielding an active area of $12.3 \times 12.3 \mathrm{~mm}^{2}$. Using a telephoto lens system the camera was able to capture a field of view (FOV) of $\sim 3.8 \times 3.8 \mathrm{~mm}^{2}$. The magnification was 
measured by placing a graduated target at the plasma position which resulted in a value of 3.2X. The temporal resolution was determined by the minimum gate width of $3 \mathrm{~ns}$. The ICCD was synchronised with a Surelite III laser using a Stanford DG 535 delaygate generator with a total trigger jitter of less than $1 \mathrm{~ns}$ which was verified using a fast sampling digital oscilloscope. For all experiments we define the time delay to be the time interval following the peak of the plasma producing laser pulse. Emission from $\mathrm{Al}^{+}$ions was selected by means of a narrow bandpass filter (FWHM $10 \mathrm{~nm}$ ) centred at $460 \mathrm{~nm}$ which isolated line emission due to transitions from $\mathrm{Al}^{+}$ions only. An edge pass filter was used to select radiation extending from $300 \mathrm{~nm}-950 \mathrm{~nm}$ for the broadband imaging measurements and which also served to block emission above $950 \mathrm{~nm}$, and hence any stray $1064 \mathrm{~nm}$ laser light. Images of the spectrally resolved $\mathrm{Al}^{+}$ions were obtained by averaging 10 laser shots whereas broadband images comprised single shots only, in order to avoid saturation of the ICCD camera.

The interferometer was of the Nomarski type [24] based on the manipulation of the polarisation of the probe laser beam. The experimental setup is shown in Figure 2. It is composed of two synchronised neodymium-doped yttrium aluminium garnet (Nd:YAG) lasers, one to generate the seed plasmas and the other to probe either the colliding (seed) plumes or the stagnation layer (as here) at any time after plasma or layer formation. The probe laser was operated at $532 \mathrm{~nm}$ and a $4 \mathrm{~ns}$ FWHM.

For Nomarski interferometry the two lasers and a complimentary metal-oxide semiconductor (CMOS) camera (C Cam Ltd, Model: BCi4) were synchronised using two Stanford DG535 delay generators which resulted in a maximum temporal jitter of 1 ns. This was verified and monitored online during the experiment using fast photodiodes and a fast sampling digital oscilloscope. A TTL master pulse was sent to trigger the Surelite III laser to create the seed plasmas. The probe laser delay was scanned using the delay generators to interrogate the stagnation layer at different times before, during and after its formation. The camera was triggered synchronously with the probe laser beam and the shutter was left open for $1 \mu \mathrm{s}$ during which time it captured the $4 \mathrm{~ns}$ duration interferogram. A $532 \mathrm{~nm}$ bandpass filter was placed in front of the camera to ensure that only the light from the interferometer laser was detected by the CMOS chip.

\section{Results and Discussions}

\subsection{Time and Spectrally Resolved Fast Photography}

Time resolved optical imaging of laser produced plasmas with fast framing cameras has been a widely used diagnostic for several years [23, 25]. Our focus here is specifically to compare the spatio-temporal evolution of ions, here $\mathrm{Al}^{+}$(spectrally resolved), and the broader plasma evolution (broadband emission), at and beyond stagnation, with that of the electrons.

The strongest transitions lying within the bandwidth of the $460 \mathrm{~nm}$ filter belong 
to the $3 \mathrm{~s} 4 \mathrm{~d}\left({ }^{3} \mathrm{D}\right)-3 \mathrm{~s} 7 \mathrm{f}\left({ }^{3} \mathrm{~F}\right)$ transition array of the $\mathrm{Al}^{+}$spectrum. The upper ${ }^{3} \mathrm{~F}$ and ${ }^{3} \mathrm{D}$ levels lie 17.76 and $15.06 \mathrm{eV}$ above the ground $3 \mathrm{~s}^{2}\left({ }^{1} \mathrm{~S}\right)$ state of $\mathrm{Al}^{+}$respectively. A recurring issue in spectroscopy and spectrally resolved imaging of laser produced plasmas is opacity, which in extreme cases, can result in self reversal of the emission line or lines under consideration [26]. The images presented here are free from such effects and we have verified this in a separate experiment where we have measured the time and space resolved spectra. We have measured here the $\mathrm{Al}^{+}$luminous front expansion with time along paths normal, at 45 degrees and parallel to the flat target surface in order to obtain an appropriately representative range of the singly charged ion velocities in the expanding seed plasmas. The luminous front is defined to be the position of the plasma front where the emission is measured to be $10 \%$ of the peak plasma emission. These paths are drawn in figure 3a for the image recorded some 30 ns after the peak of the laser pulse. The plume expansion in all three directions is shown in figure $3 \mathrm{~b}$. The values we obtain are $2.2 \pm 0.1 \times 10^{6} \mathrm{cms}^{-1}, 1.5 \pm 0.1 \times 10^{6} \mathrm{cms}^{-1}$ and $1.2 \pm$ $0.1 \times 10^{6} \mathrm{cms}^{-1}$ respectively. The fastest singly charged ions reach the collision front more quickly and their velocity can be estimated by noting that the stagnation layer starts to form and heat up $\sim 20 \mathrm{~ns}$ after the seed plasmas begin to glow. As the ions travel a distance (to the centre of the layer) of $c a .1 \mathrm{~mm}$, this group of ions has a peak velocity of $4.0 \times 10^{6} \mathrm{cms}^{-1}$. However, interrogating the broadband images, it seems that the broader plasma stagnation is observed to begin at a time delay of $15 \mathrm{~ns}$ which is likely evidence of stagnation of more highly charged ions which are known to have higher velocities than lowly charged ions and neutral atoms [27, See Figure 4, early times]. Thus some of the emission from the $\mathrm{Al}^{+}$ions at time delays $20 \mathrm{~ns}$ may be due to recombination processes. Nevertheless, we do observe space charge separation where fast electrons released promptly from the seed plasmas create an ambipolar electric field which consequently accelerates the more highly charged ions first followed by more lowly charged ions. This electron-ion separation is the main point of interest for us.

\subsection{Nomarski Interferometry}

For comparison with the spatio-temporal distributions of the singly charged ions and the broader plasma plume species at stagnation we were particularly interested in tracking the behaviour of the electrons since they could have an important role to play as progenitors in ion stagnation layer formation. Before discussing the results we outline the basic operation of the Nomarski interferometer and how it may be used to extract electron density maps.

The electron density gradient in a laser produced plasma induces a fringe shift, $\Phi$, in the interferograms which is recorded by a CCD camera. The phase shift reconstructions were obtained from the raw interferograms using a home made software package based on the Interferogram Analysis by Continuous wavelet transform Ridge Extraction (IACRE) [28]. The electron density profile of the plasma can therefore be calculated using the widely accepted standard method of Abel analysis. To extract the electron density 
profile for a relative fringe shift $\Phi$ we use the Abel equation [29]:

$$
n_{e} \cong-\lambda_{\mu m} \mathrm{n}_{c} \int_{r}^{r_{\max }} \frac{d \Phi}{d x}\left(x^{2}-r^{2}\right)^{-\frac{1}{2}} d x
$$

where, $\mathrm{n}_{e}$ is the electron density, $\lambda_{\mu m}$ is the wavelength of the probe laser in micrometers, $\mathrm{n}_{c}$ is the critical electron density and $\mathrm{x}$ and $\mathrm{r}$ are the plasma coordinates as defined in insert a) of Figure 2. The Abel inversion was performed using a home made software package using an algorithm based on Fourier Analysis [30]. Both the phase extraction software and Abel inversion software was extensively tested and benchmarked using various published interferograms and analysis in the literature.

In Figure 4 we show a series of stagnation layer interferograms and corresponding 2D electron density maps for six time delays ranging from 20 to $80 \mathrm{~ns}$. In performing this analysis we have assumed that the plasma is distributed uniformly around an axis normal to the target. We believe that we are justified in this assumption having performed fast photography in the broadband regime at angles of $0^{\circ}, 45^{\circ}$ and $90^{\circ}$ to the axis normal to the target surface. The results show the plasma emission to be symmetric to a high degree about this axis. We also assume left-right symmetry about a plane perpendicular to both the target surface and the probe beam axis. We believe this assumption is justified since we have extracted the raw phase shift map from the interferograms and taken lineouts at different points in the phase shift map which displays no significant departure from axial symmetry. In addition we have assumed that only free electrons contribute to the refractive index of the plasma. This assumption is justified by the fact that there are no transitions in $\mathrm{Al}$ atoms or ions within $0.4 \mathrm{~nm}$ of the probe laser beam wavelength [31]. Finally, the density scales on each image are chosen to best illustrate the general features of each frame. Comparisons on an absolute scale are made later. We determine the error associated with the electron density by finding the minimum detectable fringe shift and extracting its corresponding electron density. We have obtained a total value of $0.44 \times 10^{19} \mathrm{~cm}^{-3}$ for the minimum detectable fringe shift.

What is immediately clear from the image sequence is that the electrons form tightly confined structures reminiscent of ion stagnation layers at the collision front between the two seed plasma plumes and are observed to first form at a time delay of $10 \mathrm{~ns}$. At 20 ns the fringe shift pattern and corresponding electron density distribution is quite confined but also reasonably uniform. One can see that the fringe pattern is pinched close to the target at a position where it is sandwiched directly between the two seed plasmas. The electron density is greatest at a distance of approximately $0.4 \mathrm{~mm}$ from the target surface where it reaches a peak value in excess of $4 \pm 0.22 \times 10^{19} \mathrm{~cm}^{-3}$ at a time delay of $40 \mathrm{~ns}$ (see Figure 5). The electron density subsequently begins to diminish from that point on, so that by 80 ns its peak value has dropped significantly and the distribution has flattened somewhat.

In Figure 5 we plot the evolution of the electron density profile at a distance of $0.4 \mathrm{~mm}$ from the target surface for delay times ranging from 10 - 80 ns after the peak of the seed plasma laser. The first detectable fringe shift occurs at a delay time of $10 \mathrm{~ns}$ following the creation of the seed plasmas. As time proceeds, a soft electron 
stagnation (e.g. $10 \mathrm{~ns}$ frame) followed by rapid further stagnation, which peaks at $40 \mathrm{~ns}$ or so, is observed. After that the electron layer becomes broader and more damped. As noted above, by 80 ns the peak electron density has dropped to a value in the low $10^{19} \mathrm{~cm}^{-3}$ range and soon after that drops further to leave a residual density below our minimum detectable value. It is instructive to make a direct comparison of the ion spatial distribution with the electron distribution and we do so in Figure 6, where we show a 2-D spatially and temporally resolved montage of electron density, $\mathrm{Al}^{+}$ionic and broadband optical emission distributions. The density (upper row) and intensity (lower rows) scales are the same for each panel to aid direct comparison. One can see a broad, long and weakly stagnating layer in the electron density distribution at $10 \mathrm{~ns}$ resulting from a fringe shift induced by electron localisation at this time delay.

The broadband images display evidence of nascent stagnation at $15 \mathrm{~ns}$. The singly charged ions are observed to stagnate in the vicinity of the collision front at a delay time of $20 \mathrm{~ns}$ or approximately $10 \mathrm{~ns}$ after that of the electrons and $5 \mathrm{~ns}$ after the epoch of first broadband plasma emission from the collision front. The broadband images comprise superposed emission from a range of ion stages and so will be characteristic of the plasma as a whole. We attribute these observations to charge separation in the seed plasma plumes. Immediately after the seed plasmas are formed, the fast electrons try to move toward the mid-plane leaving behind the heavier ions, thus creating a space charge potential (otherwise know as an ambipolar potential and associated field). This field impresses a corresponding acceleration on each ion depending on its charge. For this reason more highly charged ions will acquire a higher velocity at early times. There is already some corroboraing evidence that space charge separation occurs in expanding plasma plumes produced by 'long' laser pulses (> 100 ps). For example Okano et al.) [19] infer space charge separation to explain the results of a time resolved electron shadowgraphy measurements on an expanding copper plasma plume. In our case, by causing these plumes to collide and using both 'ion and electron imaging', we have shown this separation optically.

Coming back to our results, as the electron front leads the ion front one expects stagnation of the electrons to occur before that of the ions and indeed the stagnation of more highly charged ions before that of the lower charged ions. Of course any electronion separation is mediated by the ambipolar field which will limit it to a dimension less than the Deybe length, $\lambda_{D}$, which will be on average $\sim 100 \mu \mathrm{m}$ in our case. $\lambda_{D}$ for the stagnated plasma can be expected to change somewhat in space and time as it evolves but this variation will be significantly smaller than in the case of an expanding laser plasma plumes. The time delay of $10 \mathrm{~ns}$ between electron and singly charged ion stagnation is consistent with the transit time obtained by dividing the Debye length by the average ion velocity. The time delay of $5 \mathrm{~ns}$ between electron stagnation and broader plasma stagnation is also well within the limits set by the Deybe length.

We can speculate further that the early stagnation of the electrons at the mid-plane collision front induces a significant "screening" effect which permits the ions (especially more highly charged ions) to approach each other quite closely leading to a tight ion 
stagnation layer, especially at $40 \mathrm{~ns}$ where the electron density reaches quite a high value $\left(\sim 4.5 \pm 0.22 \times 10^{19} \mathrm{~cm}^{-3}\right)$. However, as noted by Wan et al. [6], a multifluid code, for example [15] including an electric field solver is needed to model this situation properly.

\section{Conclusions}

We investigated electron and ion distribution of the stagnation layer formed at the interface of two laterally colliding laser produced plasmas. The plasmas were created by splitting a $6 \mathrm{~ns}, 600 \mathrm{~mJ}$ pulse from a Nd:YAG laser into two parts and focussing them onto two spots on a flat slab of aluminum. 2-D spatially and temporally resolved interferograms of the stagnation layer were obtained, revealing the evolution of its electron density. Optical imaging with an ICCD provided 2-D maps of the spatial distribution of the ions in the stagnation layer. The electron stagnation layer is observed to have a peak electron density of $4.5 \times 10^{19} \mathrm{~cm}^{-3}$ at a delay time of $40 \mathrm{~ns}$ after the peak of the seed plasma laser pulse. Significant stagnation of the electrons is observed to occur at a probe delay time of $10 \mathrm{~ns}$, while stagnation of the broader plasma occurs at a time delay of $15 \mathrm{~ns}$ and the $\mathrm{Al}^{+}$ions are found to stagnate somewhat later at a time delay of $20 \mathrm{~ns}$ after the peak of the seed plasmas. This is attributed to the effects of space charge separation that has already been inferred to occur in at least one other experiment. The data will be very useful in helping to build up a more complete picture of the physics of colliding plasmas and especially the role of ambipolar diffusion and electron stagnation in ion layer formation.

\section{Acknowledgments}

This work was sponsored by Science Foundation Ireland Frontiers Programme under grant number PHY041 and the HEA PRTLI IV INSPIRE programme of the 2nd National Development Plan programme (NDP2). Three of the authors (PH and TJK and $\mathrm{PH}$ ) would like to acknowledge support from the Irish Research Council for Science Engineering and Technology. SSH acknowledges funding from the DCU International Visiting Fellows Programme. Work associated with EU COST Action MP0601.

\section{References}

[1] Ramsden S A, Davies W E R (1964) Phys. Rev. Lett. 13227

[2] O'Haire R, McGlynn E, Henry M O and Mosnier J-P (2007) Superlatt \&6 Microstruct. 42468

[3] Hayden P, Cummings A, Murphy N, O'Sullivan G, Sheridan P, White J and Dunne P (2006) J. Appl. Phys. 99093302

[4] Bulanov S V, Esirkepov T Z, Kamenets F F, Kato Y, Kuznetsov AV, Nishihara K, Pegoraro F, Tajima T, Khoroshkov V S (2002) Plasma Phys. Rep. 28, 975

[5] Rumsby P T, Paul J W M and Masoud M M (1974) Plasma Phys. Controlled Fusion. 16969.

[6] Wan A S, Barbee T W, Cauble R, Celliers P, Da Silva L B, Moreno J C, Rambo P W, Stone G F, Trebes J E and Weber F (1997) Phys. Rev. E. 556293 
[7] Farley D R, Estabrook K G, Glendinning S G, Glenzer S H, Remington B A, Shigemori K, Stone J M, Wallace R J, Zimmerman G B and Harte J A (1999) Phys. Rev. Lett. 831982

[8] Chenaispopovics C, Rancu O, Renaudin P, Kawagoshi H, Gauthier J C, Dirksmoller M, Uschmann I, Missalla T, Forster E, Renner O, Krousky E, Pepin H, Larroche O, and Peyrusse O (1995) J. Quant. Spect. \&3 Rad. Trans. 54105

[9] Babushok V I, DeLucia F C, Gottfried J L, Munson C A, Miziolek A W (2006) Spectrochimica Acta Part B. 61999

[10] Murphy A, Hirsch J S, Kilbane D, Kennedy E T, Khater M A, Mosnier J-P, Neogi A, O'Sullivan G, Lewis C L S, Topping S, Clarke R, Divall E, Foster P, Hooker C, Langley A, Neely D, Dunne P, and Costello J T (2003) Proc SPIE 48781196

[11] Dunne P, O'Sullivan G, and O'Reilly D (2000) Appl. Phys. Lett. 7634

[12] Remington B A, Haan S W, Glendinning S G, Kilkenny J D, Munro D H and Wallace R J (1992) Phys. Fluids B 44 967-978

[13] Back C A, Glenzer S H, Landen O L, MacGowan B J and Shepard T D (1997) Rev. Sci. Instrum. $681831-833$

[14] Smith R A, Lazarus J, Hohenberger M, Marocchino A, Robinson J A, Chittenden J P, Moore A S, Gumbrell E T and Dunne (2007) Plasma Phys. Control. Fusion 49 B117-B124

[15] Rambo P W, and Denavit J (1994) J. Phys. Plasmas 124050

[16] Bosch R A, Berger R L, Failor B H, Delamater N D, Charatis G and Kauffman G (1992) Phys. Fluids B 44 979-988

[17] Luna H, Kavanagh K D, and Costello J T (2007) J. Appl. Phys. 101033302

[18] Pollaine S M, Berger R L, Keane C J (1992) Phys. Fluids B 44 989-991

[19] Okano Y, Hironaka Y, Nakamura K G, and Kondo K-I (2003) Appl. Phys. Lett. 838

[20] Purvis M, Grava J, Filevich J, Marconi M C, Dunn J, Moon S J, Shlyaptsev V N, Jankowska E, and Rocca J J (2007) Phys. Rev. E. 76046402

[21] Smith R A, Lazarus L, Hohenberger M, Moore A S, Robinson J S, Gumbrell E T, Dunne M (2007) Astrophys.Space Sci. $\mathbf{3 0 7} 131$

[22] Harilal S S, Bindhu C V, Kunze H-J (2001) J. Appl. Phys. 894737

[23] Doria D, Kavanagh K D, Costello J T, and Luna H (2006) Meas. Sci. Technol. 17670

[24] Benattar R, Popovics C and Sigel R (1979) Rev. Sci. Instrum. 501583

[25] Harilal S S, O'Shay B, Tillack M S, Bindhu C V, and Najmabadi F (2005) IEEE Trans. Plasma Sci. 32474

[26] Sakka T, Nakajima T and Ogata Y H (2002) J. Appl. Phys. 925 2296-2303

[27] Hirsch J S, Meighan O, Mosnier J-P, van Kampen P, Whitty W W, Costello J T, Lewis C L S, MacPhee A G, Hirst G J, Westhall J, and Shaikh W (2000) J. Appl. Phys. 889

[28] Tomassini P, Giulietti A, Gizzi L A, (2001) App. Opt. 4035 6561-6568

[29] Ruiz-Camacho J, Beg F N and Lee P (2007) J. Phys. D: Appl. Phys. 402026

[30] Pretzler G, (1991)Zeitschrift Fur Natureforschung 467

[31] National Institute of Standards and Technology. http://physics.nist.gov/PhysRefData/ASD/ 
Figure 1. Setup to create the colliding laser produced plasmas. The incoming laser beam from a Surelite III Nd:YAG laser is split into two beams by a wedge prism and focused to two points using a plano convex lens. The target is orientated at $45^{\circ}$ to the laser pulse.

Figure 2. Schematic diagram of interferometer experimental setup. A $1064 \mathrm{~nm}$ wavelength laser pulse with an energy of $600 \mathrm{~mJ}$ (FWHM $6 \mathrm{~ns}$ ) is used to create the colliding laser produced plasma plumes. A frequency doubled $(532 \mathrm{~nm}) \mathrm{Nd}$-YAG laser (FWHM $4 \mathrm{ns)}$ ) is used as the light source for the interferometer. The interferometer is of the Nomarski polarisation type. Insert a): definition of plasma coordinate system.

Figure 3. Top: $\mathrm{Al}^{+}$image for one time delay showing trajectories chosen to determine seed plume expansion velocities. Bottom: Plume front positions as a function of time, parallel and at $45^{\circ}$ to the target surface.

Figure 4. Sequence of optical interferograms showing the temporal and spatial evolution of the electron density in the stagnation layer created at the interface between two colliding laser produced plasma plumes.

Figure 5. Evolution of the stagnation layer electron density profile at a distance of $0.4 \mathrm{~mm}$ from the target surface for different time delays.

Figure 6. 2-D temporally and spatially resolved electron density and ion distribution maps. The top panels show the 2D electron density profile in the stagnation region between the two seed plasmas at time delays of 10, 15 and $20 \mathrm{~ns}$. The centre panels show the $\mathrm{Al}^{+}$ion emission in the corresponding region at the same times and bottom panel shows the corresponding broadband emission.

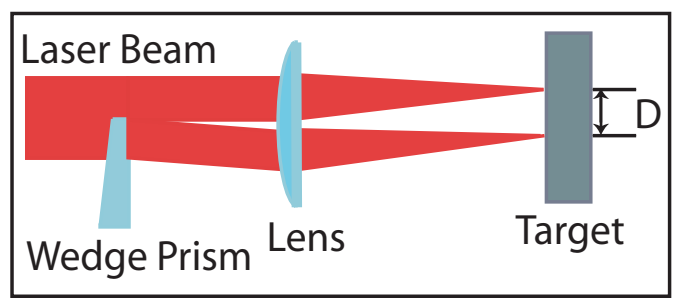




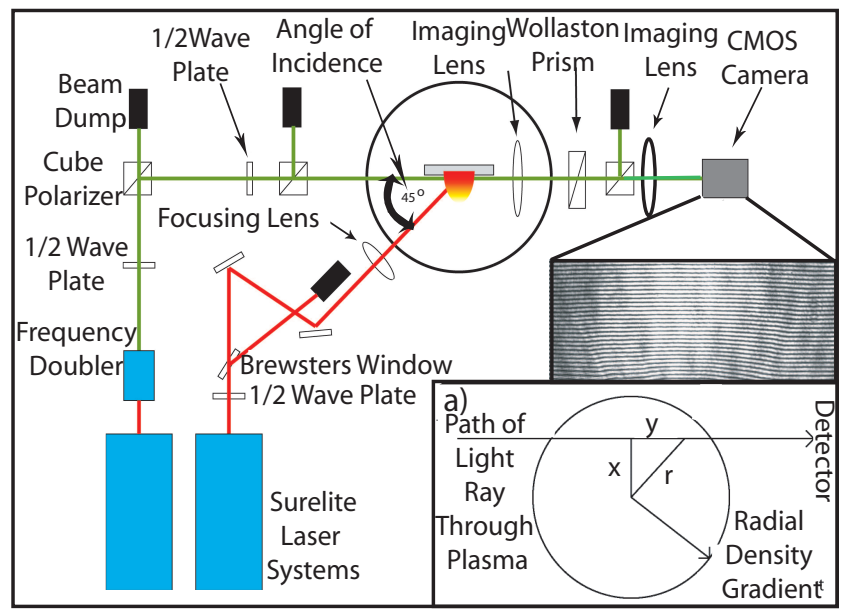



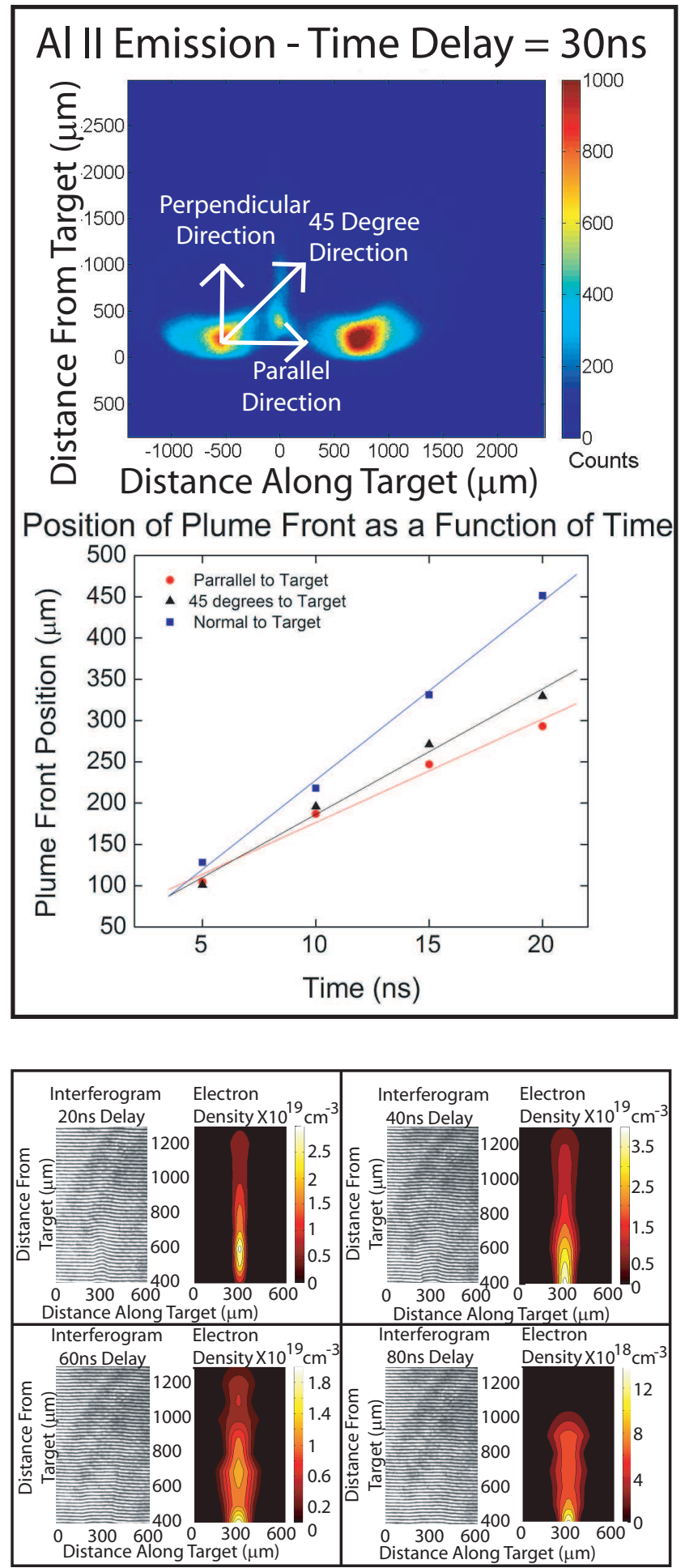


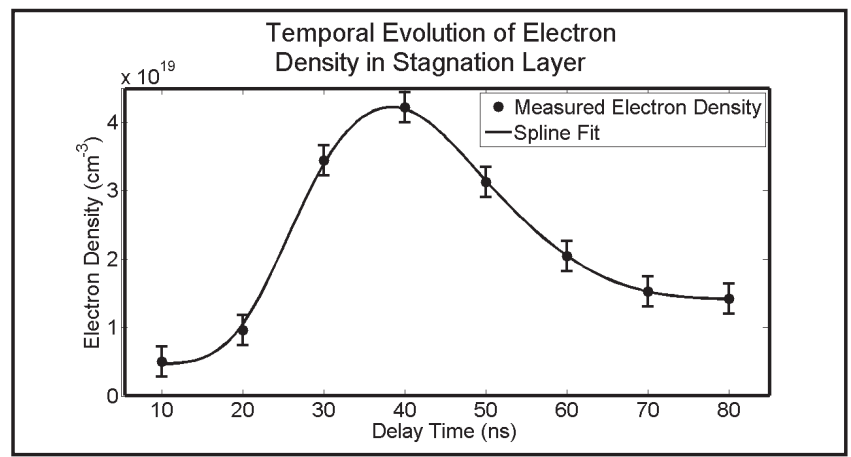

\begin{tabular}{|c|c|c|}
\hline 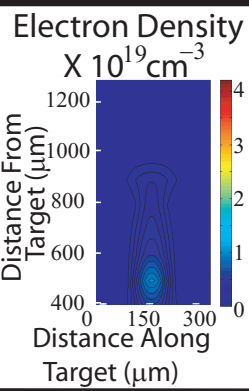 & $\begin{array}{l}\text { Electron Density } \\
\times 10^{19} \mathrm{~cm}^{-3} \\
{ }^{4} \\
{ }^{1200} \\
\\
\text { Distance Along } \\
\text { Target }(\mu \mathrm{m})\end{array}$ & 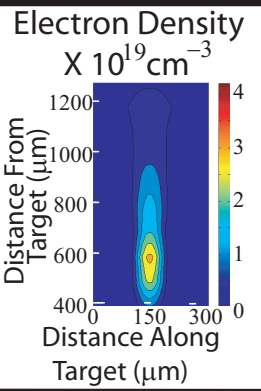 \\
\hline 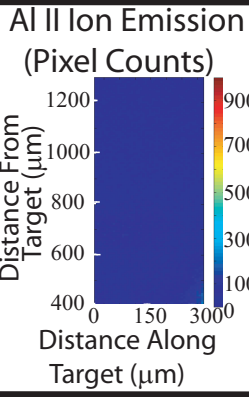 & 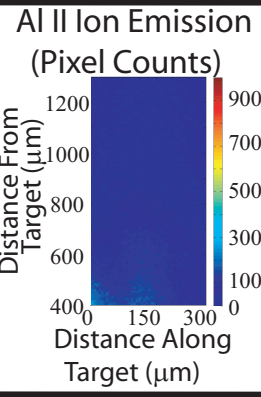 & 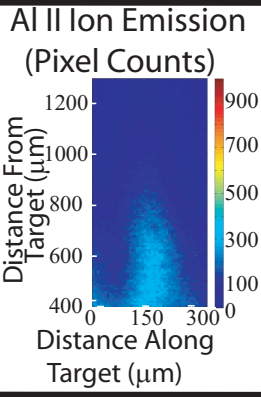 \\
\hline $\begin{array}{c}\text { Broadband } \\
\text { Emission } \\
\text { (Pixel Counts) } \\
\text { (Pixel } \\
10 \text { ns Time Delay } \\
\text { Target ( } \mu \text { m) } \\
\text { Distance Along } \\
\text { non }\end{array}$ & 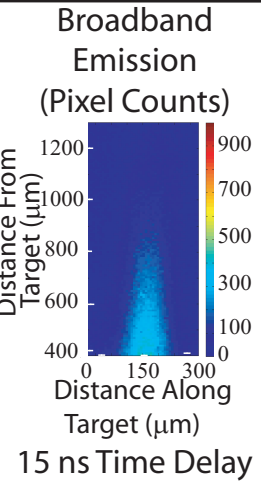 & $\begin{array}{c}\text { Broadband } \\
\text { Emission } \\
\text { (Pixel Counts) } \\
\text { (Pix) } \\
\text { Target ( } \mu \mathrm{m} \text { ) } \\
20 \text { ns Time Delay }\end{array}$ \\
\hline
\end{tabular}

